\title{
ANALYTICAL RELATIONSHIPS BETWEEN BULK MICROPHYSICAL PARAMETERS OF NUCLEATION, AITKEN, ACCUMULATION AND COARSE MODE PARTICLES AND EXTINCTION AND BACKSCATTER COEFFICIENTS MEASURED WITH LIDAR

\author{
Alexei Kolgotin ${ }^{1^{*}}$, Detlef Müller ${ }^{2}$, Vadim Griaznov ${ }^{1}$, \\ Igor Veselovskii ${ }^{1}$, Mikhail Korenskiy ${ }^{1}$, Albert Ansmann ${ }^{3}$ \\ (l) A.M. Prokhorov General Physics Institute, Moscow, Russia, Email*: kolgotin@pic.troitsk.ru \\ ${ }^{(2)}$ University of Hertfordshire, Hatfield, Hertfordshire, AL10 9AB, UK \\ ${ }^{(3)}$ Leibniz Institute for Tropospheric Research, Leipzig, Germany
}

\begin{abstract}
We derive analytical relationships between bulk microphysical parameters of nucleation, Aitken, accumulation and coarse mode particles and extinction and backscatter coefficients measured at wavelengths 355, 532 and $1064 \mathrm{~nm}$. The bulk parameters are represented by number concentration, mean (effective) radius, variance and complex refractive index. Analytical relationships hold true for arbitrarily shaped particles and complex refractive indices $m=m_{\mathrm{R}}-i m_{\mathrm{I}}$, where $m_{\mathrm{R}}>>m_{\mathrm{I}}$. The accuracy is above $60 \%$.
\end{abstract}

\section{INTRODUCTION}

Retrieval of particle microphysical parameters from lidar measurements of extinction coefficients at 355 and $532 \mathrm{~nm}$ and backscatter coefficients at 355, 532 and $1064 \mathrm{~nm}$ is a complex task which can be solved in terms of parametric and direct, inversion (as, for example, least-squares approaches) and inversion with regularization methods. Direct methods of solving this task became popular recently. The direct approach uses a sample function, as for example a logarithmicnormally distributed function that approximates atmospheric particle size distributions.

The most common case permits the use of up to 5 aerosol components [1]. In this contribution we further simplify this concept. We describe an arbitrary external mixture as superposition of 4 components, i.e. particles in the nucleation, Aitken, accumulation and coarse mode, respectively. We present standard algebraic equations that describe the interdependencies between backscatter and extinction coefficients and particle bulk parameters, i.e. number concentration, mean (effective) radius, variance and complex refractive index for each mode separately.

In section 2 we present the methodology. In section 3 we carry out a numerical test with synthetic optical data. Section 4 summarizes our results.

\section{METHODOLOGY}

Extinction $\alpha(\lambda)$ and backscatter $\beta(\lambda)$ coefficients measured with lidar at wavelengths $\lambda=355,532$ and $1064 \mathrm{~nm}$ are related to the particle size distribution $f(r)$ via integral equations of the form

$$
\int_{0}^{\infty} K_{g}(\lambda, m, r) f(r) d r=g(\lambda), \quad g=\alpha, \beta
$$

where the kernels of the integral equation $K_{g}(\lambda, m, r)$ are extinction $(g=\alpha)$ or backscatter $(g=\beta)$ cross-sections of particles of radius $r$ and complex refractive index $m=m_{\mathrm{R}}-i m_{\mathrm{I}}$. The real and imaginary parts of the complex refractive index fulfill the conditions

$$
m_{\mathrm{R}} \in[1.3,1.8] \quad m_{\mathrm{I}} \in[0,0.1]
$$

For example, if the function $f(r)$ describes number particle size distribution we can compute mean (effective) radius $r_{\mathrm{m}}\left(r_{\text {eff }}\right)$, standard deviation $\sigma$, number $(N)$, surface-area $(S)$ and volume $(V)$ concentrations. We can split the radius domain $r \in(0 ; \infty)$ into 4 intervals [in $\mu \mathrm{m}$ ]

$$
\begin{array}{llll}
r \in(0,0.005) & {[\mathrm{a}]} & r \in(0.005,0.1) & {[\mathrm{b}]} \\
r \in(0.1,0.5) & \text { [c] } & r \in(0.5, \infty) & \text { [d] (3) }
\end{array}
$$

and formally consider Eq. (1) on each interval separately. This approach leads us to the representation of optical data as a superposition of optical data of the nucleation [Nu, interval (3a)], Aitken [Ai, (3b)], accumulation [Ac, (3c)] and coarse $[\mathrm{Co},(3 \mathrm{~d})]$ mode particles, i.e.

$$
g_{\mathrm{Nu}}(\lambda)+g_{\mathrm{Ai}}(\lambda)+g_{\mathrm{Ac}}(\lambda)+g_{\mathrm{Co}}(\lambda)=g(\lambda) \quad g=\alpha, \beta(4)
$$

We aim to express the optical data $g(\lambda)$ of each mode $f_{\mathrm{mo}}(r)$ on the basis of its own particle microphysical parameters $\mu_{\text {mo }}\left(r_{\text {eff,mode }}\right), \sigma_{\text {mo }}, N_{\text {mo }}$ and $m_{\text {mo }}$ where each number mode is approximated by a lognormal distribution, i.e.

$$
\begin{gathered}
f_{\mathrm{mo}}(r) \approx \frac{N_{\mathrm{mo}}}{(2 \pi)^{1 / 2} r \ln \sigma_{\mathrm{mo}}} \exp \left[-\frac{\left(\ln r-\ln \mu_{\mathrm{mo}}\right)^{2}}{2\left(\ln \sigma_{\mathrm{mo}}\right)^{2}}\right] \\
\mathrm{mo}=\mathrm{Nu}, \mathrm{Ai}, \mathrm{Ac}, \mathrm{Co}
\end{gathered}
$$

\subsection{Case of large particles (coarse mode)}

We start our study with the largest particles. In that case $r \rightarrow \infty$. In this asymptotic case the extinction kernel $K_{\alpha}(\lambda, m, r)$ converges to the parti- 
cle cross-section $\pi r^{2}$ with factor $\sim 2.1$. As a result, the left-hand side of Eq. (1) coincides with the definition of surface-area concentration [2], i.e.

$$
\int_{0.5}^{\infty} K_{\alpha}(\lambda, m, r) f(r) d r \approx \frac{2.1}{4} S \quad g=\alpha, \beta
$$

A statistical analysis of our reference look-up table shows that extinction coefficient and surface-area concentration of large particles are linearly correlated with a high correlation coefficient close to 1 [2]. For particles with effective radius $r_{\text {eff }} \geq 0.5 \mu \mathrm{m}$ and measured with lidar at $\lambda=532 \mathrm{~nm}$ the equation

$$
\alpha_{\text {Co }}(532)=0.635 S \pm 25 \%
$$

is fulfilled. In view of approximation (5) this equation can be written in terms of number concentration, mean (effective) radius and variance $\alpha_{\mathrm{Co}}(532)=$

$$
\begin{aligned}
& =0.635 \times 4 \pi N_{\mathrm{Co}} r_{\mathrm{eff}, \mathrm{Co}}{ }^{2} \exp \left(-3 \ln ^{2} \sigma_{\mathrm{Co}}\right) \pm 25 \% \\
& =0.635 \times 4 \pi N_{\mathrm{Co}} \mu_{\mathrm{Co}}^{2} \exp \left(2 \ln ^{2} \sigma_{\mathrm{Co}}\right) \pm 25 \%
\end{aligned}
$$

Eq. (8) can be directly used for the analytical estimation of the coarse-mode extinction coefficient from particle microphysical parameters and conversely. The estimation accuracy $\varepsilon_{\mathrm{Co}}= \pm 25 \%$ in Eq. (8) is defined as

$$
\begin{gathered}
\varepsilon_{\mathrm{mo}}(\lambda)=g_{\text {true }}(\lambda) / g_{\mathrm{mo}}(\lambda)-1 \quad g=\alpha, \beta ; \\
\mathrm{mo}=\mathrm{Nu}, \mathrm{Ai}, \mathrm{Ac}, \mathrm{Co},
\end{gathered}
$$

wherer term $g_{\text {true }}(\lambda)$ is the true value. Eq. (8) neither depends on particle shape nor complex refractive index.

\subsection{Nucleation mode and Aitken mode}

We now consider the smallest particles $r_{\text {lim }}<0.1$ $\mu \mathrm{m}$. In this case the dimensionless size parameter is $x_{\lim }=2 \pi r_{\lim } / \lambda \approx 1$ at $\lambda=532 \mathrm{~nm}$. We need to use the $1^{\text {st }}$ and $2^{\text {nd }}$ member of the series expansion that asymptotically approximates the kernels $K_{g}(\lambda, m, r)$ in Mie theory. The use of the $2^{\text {nd }}$ member of the series expansion provides us with a sufficiently accurate approximation, if the magnitude $x_{\lim } \approx 1 \quad[3]$. This $2^{\text {nd }}$ order approximation allows us to find the kernels $K_{g}(\lambda, m, r)$ and one can show that

$K_{\alpha}(\lambda, m, r)=A_{1}(\lambda, m) r^{3}+A_{2}(\lambda, m) r^{5}+A_{3}(\lambda, m) r^{6}(10)$

$K_{\beta}(\lambda, m, r)=B_{1}(\lambda, m) r^{6}+B_{2}(\lambda, m) r^{8}+B_{3}(\lambda, m) r^{10}(11)$ where

$$
\begin{gathered}
A_{1}(\lambda, m)=-\frac{8 \pi^{2}}{\lambda} \operatorname{Im}\left(\frac{m^{2}-1}{m^{2}+2}\right) ; \\
A_{2}(\lambda, m)=-\frac{32 \pi^{4}}{15 \lambda^{3}} \operatorname{Im}\left[\left(\frac{m^{2}-1}{m^{2}+2}\right)^{2} \frac{m^{4}+27 m^{2}+38}{2 m^{2}+3}\right] ; \\
A_{3}(\lambda, m)=-\frac{128 \pi^{5}}{3 \lambda^{4}} \operatorname{Re}\left[\left(\frac{m^{2}-1}{m^{2}+2}\right)^{2}\right] ; \\
B_{1}(\lambda, m)=B_{1}\left(\lambda, m_{\mathrm{R}}\right)=\left(\frac{2 \pi}{\lambda}\right)^{4}\left(\frac{m_{\mathrm{R}}{ }^{2}-1}{m_{\mathrm{R}}{ }^{2}+2}\right)^{2} ;
\end{gathered}
$$

$$
\begin{gathered}
B_{2}(\lambda, m)=B_{2}\left(\lambda, m_{\mathrm{R}}\right)=2\left(\frac{2 \pi}{\lambda}\right)^{6}\left(\frac{m_{\mathrm{R}}{ }^{2}-1}{m_{\mathrm{R}}{ }^{2}+2}\right)^{2} \kappa\left(m_{\mathrm{R}}\right) ; \\
B_{3}(\lambda, m)=B_{3}\left(\lambda, m_{\mathrm{R}}\right)=\left(\frac{2 \pi}{\lambda}\right)^{8}\left(\frac{m_{\mathrm{R}}{ }^{2}-1}{m_{\mathrm{R}}{ }^{2}+2}\right)^{2} \kappa^{2}\left(m_{\mathrm{R}}\right) ; \\
\kappa(m)=\kappa\left(m_{\mathrm{R}}\right)=\frac{3}{5} \frac{m_{\mathrm{R}}{ }^{2}-2}{m_{\mathrm{R}}{ }^{2}+2}-\frac{1}{30}\left(m_{\mathrm{R}}{ }^{2}+2\right)-\frac{5}{30} \frac{m_{\mathrm{R}}{ }^{2}+2}{2 m_{\mathrm{R}}{ }^{2}+3}
\end{gathered}
$$

The equalities $B_{j}(\lambda, m)=B_{j}\left(\lambda, m_{\mathrm{R}}\right)$ in Eq. (13) hold true because $m_{\mathrm{R}}>>m_{\mathrm{l}}$, see the conditions (2).

We now come back to Eq. (1) in which the kernels $K_{g}(\lambda, m, r)$ are determined with Eqs. (10) and (11). Eq. (1) can be analytically integrated if we use approximation (5) for the function $f(r)$ [4]

$$
\begin{array}{r}
\alpha_{\mathrm{mo}}(\lambda)=N_{\mathrm{mo}}\left[A_{1}(\lambda, m) \mu_{\mathrm{mo}}{ }^{3} \exp \left(4.5 \ln ^{2} \sigma_{\mathrm{mo}}\right)+\right. \\
A_{2}(\lambda, m) \mu_{\mathrm{mo}} \exp \left(12.5 \ln ^{2} \sigma_{\mathrm{mo}}\right)+ \\
\left.A_{3}(\lambda, m) \mu_{\mathrm{mo}}{ }^{6} \exp \left(18 \ln ^{2} \sigma_{\mathrm{mo}}\right)\right] \\
\beta_{\mathrm{mo}}(\lambda)=N_{\mathrm{mo}}\left[B_{1}\left(\lambda, m_{\mathrm{R}}\right) \mu_{\mathrm{mo}}{ }^{6} \exp \left(18 \ln ^{2} \sigma_{\mathrm{mo}}\right)+\right. \\
B_{2}\left(\lambda, m_{\mathrm{R}}\right) \mu_{\mathrm{mo}}{ }^{8} \exp \left(32 \ln ^{2} \sigma_{\mathrm{mo}}\right)+ \\
\left.B_{3}\left(\lambda, m_{\mathrm{R}}\right) \mu_{\mathrm{mo}}{ }^{10} \exp \left(50 \ln ^{2} \sigma_{\mathrm{mo}}\right)\right] \\
\mathrm{mo}=\mathrm{Nu}, \mathrm{Ai}
\end{array}
$$

The estimation accuracies that follow for the extinction and backscatter coefficients with these analytical expressions are close to $\varepsilon_{\text {mode }}=0$ for nucleation and Aitken modes if $f_{\text {mo }}\left(\mu_{\text {mo }}\right) \gg>f_{\text {mo }}\left(r_{\text {lim }}\right)=f_{\text {mo }}(0.1)$.

Eqs. (14) and (15) still can be used with an accuracy up to $\varepsilon_{\mathrm{mo}} \approx 15 \%$ if $\mu_{g} \leq r_{\mathrm{lim}}=0.1 \mu \mathrm{m}$ where

$$
\mu_{g}=\frac{\int_{0}^{\infty} r K_{g}(\lambda, m, r) f_{\mathrm{mo}}(r) d r}{g_{\mathrm{mo}}(\lambda)}=\frac{M_{g, \mathrm{mo}}(\lambda, m)}{g_{\mathrm{mo}}(\lambda)}
$$

and

$$
\begin{gathered}
M_{\alpha, \mathrm{mo}}(\lambda, m)=N_{\mathrm{mo}}\left[A_{1}(\lambda, m) \mu_{\mathrm{mo}}{ }^{4} \exp \left(8 \ln ^{2} \sigma_{\mathrm{mo}}\right)+\right. \\
A_{2}(\lambda, m) \mu_{\mathrm{mo}}{ }^{6} \exp \left(18 \ln ^{2} \sigma_{\mathrm{mo}}\right)+ \\
\left.A_{3}(\lambda, m) \mu_{\mathrm{mo}}{ }^{7} \exp \left(24.5 \ln ^{2} \sigma_{\mathrm{mo}}\right)\right](17) \\
M_{\beta, \mathrm{mo}}(\lambda, m)=N_{\mathrm{mo}}\left[B_{1}\left(\lambda, m_{\mathrm{R}}\right) \mu_{\mathrm{mo}}{ }^{7} \exp \left(24.5 \ln ^{2} \sigma_{\mathrm{mo}}\right)+\right. \\
B_{2}\left(\lambda, m_{\mathrm{R}}\right) \mu_{\mathrm{mo}}{ }^{9} \exp \left(40.5 \ln ^{2} \sigma_{\mathrm{mo}}\right)+ \\
\left.B_{3}\left(\lambda, m_{\mathrm{R}}\right) \mu_{\mathrm{mo}}{ }^{11} \exp \left(60.5 \ln ^{2} \sigma_{\mathrm{mo}}\right)\right](18)
\end{gathered}
$$

Standard multiwavelength lidar delivers 3 backscatter and 2 extinction coefficients. If we consider the five expressions (14) and (15) in the spectral range $\lambda=355-1064 \mathrm{~nm}$ we obtain a system of 5 nonlinear algebraic equations and 5 unknown parameters $N_{\mathrm{mo}}, \mu_{\mathrm{mo}}, \sigma_{\mathrm{mo}}$ and $m_{\mathrm{mo}}$. This system can be solved numerically, see Ref. [5], for the case of single modes and small particles in the Aitken mode.

We stress that Eq. (14) and (15) can be modified with a correction factor $r_{\text {lim }} / \mu_{g}$ so that

$$
\begin{array}{ll}
\alpha_{\mathrm{mo}}{ }^{\operatorname{corr}}(\lambda)=\left(r_{\text {lim }} / \mu_{\alpha}\right) \alpha_{\mathrm{mo}}(\lambda) & \mathrm{mo}=\mathrm{Nu}, \mathrm{Ai}(19) \\
\beta_{\mathrm{mo}}{ }^{\operatorname{corr}}(\lambda)=\left(r_{\mathrm{lim}} / \mu_{\beta}\right) \beta_{\mathrm{mo}}(\lambda) & \mathrm{mo}=\mathrm{Nu}, \mathrm{Ai}(20)
\end{array}
$$

This correction allows us to use Eq. (14) and (15) for large $\mu_{\mathrm{mo}}$ and $\sigma_{\mathrm{mo}}$ but condition $\mu_{\mathrm{g}}=1 . .5 r_{\mathrm{lim}}$ 
in Eq. (16) for these $\mu_{\mathrm{mo}}$ and $\sigma_{\mathrm{mo}}$ still holds true. In this case the estimation accuracy of the analytical expressions is better than $\varepsilon_{\mathrm{mo}} \approx 60 \%$.

In contrast to large particles, extinction and backscatter coefficients of small particles [see analytical expressions (14), (15) and (19), (20)] significantly depend on their complex refractive index. Still, these analytical expressions can be used for arbitrarily shaped particles because their size is much less than the measurement wavelengths used in lidar applications [3].

\subsection{Intermediate size of particles}

Our analysis of Eq. (8) and (14) shows that the extinction coefficient of large particles converges to their surface-area concentration; note that we can describe this parameter in terms of volume concentration normalized by effective radius, see Ref. [2]. The extinction coefficient of particles in the nucleation mode (3a) is proportional to volume concentration normalized by wavelength. The statistical analysis of our reference look-up table shows that the extinction coefficient at 532 $\mathrm{nm}$ and surface-area concentration are still linearly correlated but with a lower correlation coefficient

$$
\begin{aligned}
& \alpha_{\mathrm{Ac}}(532)=0.57 S_{\mathrm{Ac}} \pm 60 \%= \\
& \quad=0.57 \times 4 \pi N_{\mathrm{Ac}} \mu_{\mathrm{Ac}}^{2} \exp \left(2 \ln ^{2} \sigma_{\mathrm{Ac}}\right) \pm 60 \%
\end{aligned}
$$

That means we can use the linear regression equation, but the estimation uncertainty grows to $\varepsilon_{\mathrm{Ac}}= \pm 60 \%$ in the worst case. Again, we note that Eq. (21) does not depend on the complex refractive index and particle shape; see Eq. (8).

The estimation accuracies of the analytical expressions that can be used for the extinction coefficient, see (8), (14), (19) and (21) do not exceed $60 \%$. The highest uncertainties are related to particles with a radius of approximately $0.1 \mu \mathrm{m}$ which is approximately at the border between Aitken and accumulation mode particles. We can improve the accuracy of estimation if we combine the analytical expressions with best accuracies $\varepsilon_{\mathrm{mo}}$ [see, for example, Eqs. (8)] and (14)]. In fact, any lognormal distribution (5), e.g. denoted as $f_{1}$, can be approximately represented as superposition of two other lognormal distributions, i.e. $f_{2}$ and $f_{3}[6]$

$$
f_{1} \rightarrow f_{2}+f_{3}
$$

In that case the concentrations of the particle size distributions fulfill the conditions

$$
N_{1}=N_{2}+N_{3} \quad S_{1}=S_{2}+S_{3} \quad V_{1}=V_{2}+V_{3}
$$

These conditions ensure the same effective radius $r_{\text {eff }}$ as well as similar optical data $g(\lambda)$ that are produced by $f_{1}$ and by a superposition of $f_{2}$ and $f_{3}$. We consider the conditions (22) and determine preliminary ( $1^{\text {st }}$ order) estimates of the parameters $N_{1}, \mu_{1}, \sigma_{1}$ and $N_{2}, \mu_{2}, \sigma_{2}$. Details on how this step is done exactly will be provided in a publication. We find the parameters for the $3^{\text {rd }}$ distribution which can be described by the following equations

$$
\begin{gathered}
N_{3}=N_{1}-N_{2} \\
\mu_{3}=\frac{\left[N_{1} N_{3}^{-1} \mu_{1}^{2} \exp \left(2 \ln ^{2} \sigma_{1}\right)-N_{2} N_{3}^{-1} \mu_{2}^{2} \exp \left(2 \ln ^{2} \sigma_{2}\right)\right]^{0.5}}{\exp \left(\ln ^{2} \sigma_{3}\right)} \\
\sigma_{3}=\exp \left\{\left[\frac{2}{3} \ln \ldots\right]^{0.5}\right\}
\end{gathered}
$$

where ${ }^{\cdots}$ denotes

$$
\frac{N_{1} \mu_{1}^{3} \exp \left(4.5 \ln ^{2} \sigma_{1}\right)-N_{2} \mu_{2}^{3} \exp \left(4.5 \ln ^{2} \sigma_{2}\right)}{\left[N_{1} N_{3}^{-1 / 3} \mu_{1}^{2} \exp \left(2 \ln ^{2} \sigma_{1}\right)-N_{2} N_{3}^{-1 / 3} \mu_{2}^{2} \exp \left(2 \ln ^{2} \sigma_{2}\right)\right]^{3 / 2}}
$$

Thus we can always (1) fit the parameters $\left(N_{2}\right.$, $\left.\mu_{2}, \sigma_{2}\right)$ and $\left(N_{3}, \mu_{3}, \sigma_{3}\right)$ [which simultaneously fulfill Eq. (22) and (23)], (2) find respective optical data $g_{2}(\lambda)$ and $g_{3}(\lambda)$ with, for example, Eq. (8) and/or (14) and finally (3) estimate the optical data produced by the size distribution $f_{1}$ :

$g_{1}(\lambda)=g_{\mathrm{mo}}(\lambda) \approx g_{2}(\lambda)+g_{3}(\lambda) \quad \mathrm{mo}=\mathrm{Ai}, \mathrm{Ac}(24)$ The estimation accuracies of $g_{2}(\lambda)$ and $g_{3}(\lambda)$ determine the estimation accuracy of $g_{1}(\lambda)$.

\section{NUMERICAL TEST}

We compare the extinction and backscatter coefficients $g(\lambda)$ estimated with the analytical expressions (8), (14), (15), (19)-(21), (24) and computed with a Mie-scattering code [7]. Besides that comparison, we also compare the kernels $K_{g}(\lambda, m, r)$ that result from the use of Eq. (10) and (11) and which are described by the strict theory of Mie scattering [7].

We use for the comparisons lognormal size distributions with parameters $\mu \in[0.001 ; 2.5] \mu \mathrm{m}$, $\sigma \in[1.35 ; 2.55]$ and complex refractive indices in the intervals (2) to estimate the synthetic optical data. We split the parameter domain $(\mu, \sigma)$ into 5 regions (see Fig. 1b,c). We use Eq. (8) for region I, Eq. (14) and (15) for region II, Eq. (19), (20) for region III, Eq. (21) for region IV and Eq. (24) for region $\mathrm{V}$. The results of the estimated values of the kernels and effective radius are shown in Fig. 1a and $1 \mathrm{~b}$, respectively. The estimation accuracy (9) for the extinction coefficient, i.e. $\varepsilon_{\alpha(532)}$ at $\lambda=532 \mathrm{~nm}$ and $m=1.7-i 0.05$ is shown in Fig. 1c.

Fig. 1a shows that the kernels computed with the analytical expressions (dotted lines) and with the Mie-scattering code (solid lines) coincide for all particle sizes $r<0.1 \mu \mathrm{m}$. This result shows why we obtain such a high accuracy of the estimated extinction (Fig. 1c) and backscatter (not shown) 

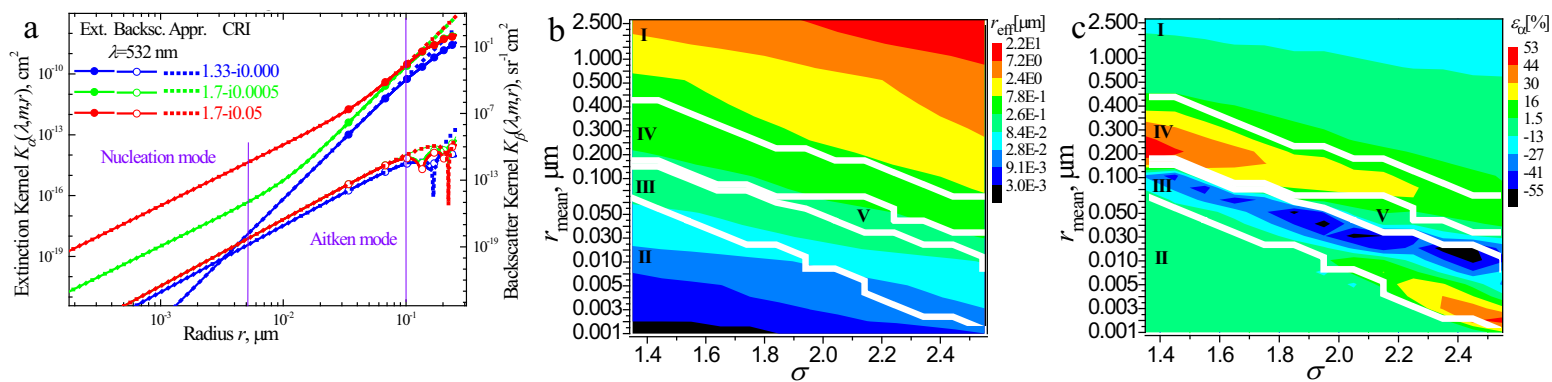

Fig. 1. (a) Kernels $K_{g}(\lambda, m, r)$ computed with the analytical expressions (dotted lines) and with a Mie-scattering code (solid lines). (b) Effective radius $r_{\text {eff }}$ and (c) estimation accuracy of the extinction coefficient $\varepsilon_{\alpha(532)}$ at $m=1.7-i 0.05$ vs mean radius and variance $\left(r_{\text {mean }}=\mu, \sigma\right)$. Regions $I$ and $I V$ are used for the correlation relationships; see text. Regions II and III are needed for the $2^{\text {nd }}$ order approximation of the kernel functions. Region V means that we use the combination of correlation (I, IV) and approximation (II-III).

coefficients, i.e. $\varepsilon_{g} \approx 0$ in region II where Eq. (14), (15) can be used. We also find a high estimation accuracy of $\left|\varepsilon_{\alpha(532)}\right| \approx 20 \%$ based on the correlation relationship used in region I. the correlation relationship (21) works, but the accuracy degrades up to $60 \%$. Region III shows an estimation accuracy of $\varepsilon_{\alpha(532)} \approx-55 \%$ (overestimation). This result indicates the limit where the use of the $2^{\text {nd }}$ order approximation of kernel functions still can be used. The region $\mathrm{V}$ where we use the combination of the correlation relationships and the $2^{\text {nd }}$ order approximation provides the accuracy to approximately $\left|\varepsilon_{\alpha(532)}\right| \approx 50 \%$.

\section{CONCLUSION}

We derive analytical expressions that allow us to estimate extinction and backscatter coefficients at wavelengths 355,532 and $1064 \mathrm{~nm}$. We derive these expressions and thus estimates of these coefficients based on the following approaches:

a. $2^{\text {nd }}$ order approximation of kernel functions can be described by Mie-scattering theory for the case of small particles with $r<0.1 \mu \mathrm{m}$ (nucleation and Aitken modes),

b. correlation relationships between extinction coefficient and surface-area concentration are used if particles are large and intermediate sizes, i.e. $r>0.1 \mu \mathrm{m}$ (accumulation and coarse modes), c. combination of approaches $\mathrm{a}$ and $\mathrm{b}$ in the case of small particles where $r \approx 0.1 \mu \mathrm{m}$ (Aitken and/or accumulation modes).

The expressions directly link the backscatter and/or extinction coefficients through the underlying particle bulk parameters such as number concentration, mean (effective) radius, variance and complex refractive index to standard algebraic equations. The expressions hold true for arbitrarily shaped particles and complex refractive indices $m=m_{\mathrm{R}}-i m_{\mathrm{I}}$, where $m_{\mathrm{R}}>>m_{\mathrm{I}}$. Minimum uncertainties of the estimates of the parameters are $0-20 \%$ in the case of nucleation and coarse mode particles, respectively. Maximum uncertainties are $60 \%$ at worst in the case of Aitken and accumulation mode particles.

We will show how these analytical expressions can be applied for estimating microphysical parameters and the contributions of nucleation, Aitken, accumulation and coarse mode particles to extinction and backscatter coefficients measured with lidar. A paper that describes the results is in preparation.

\section{REFERENCES}

1. I. Veselovskii, et al, "Characterization of smoke/dust episode over West Africa: comparison of MERRA-2 modeling with multiwavelength Mie-Raman lidar observations", Atmos. Meas. Tech., 11, pp. 949-969 (2018).

2. A. Kolgotin, et al, "Improved identification of the solution space of aerosol microphysical properties derived from the inversion of profiles of lidar optical data, part 1: theory", Appl. Opt., 55, pp. 9839 - 9849 (2016).

3. H.C. van de Hulst, Light scattering by small particles, John Wiley\&Sons, New York, 1957, $537 \mathrm{p}$.

4. I.S. Gradshtein, I.M. Ryzhik, Tables Of Integrals, Series And Products (https:// archive.org/details/GradshteinI.S.RyzhikI.M.Tab lesOfIntegralsSeriesAndProducts)).

5. A. Kolgotin, et al, "Particle microphysical parameters and complex refractive index from $3 \beta+2 \alpha$ HSRL/Raman lidar measurements: retrieval uncertainties versus measurement errors", manuscript in preparation, 2019.

6. N.C. Beaulieu, et al, "An optimal lognormal approximation to lognormal sum distributions", Transactions on Vehicular Technology, 53, No. 2, pp. 479 - 489 (2004).

7. F. B. Bohren and D. R. Huffman, Absorption and scattering of light by small particles, John Wiley, New York, 1983, 530 p. 